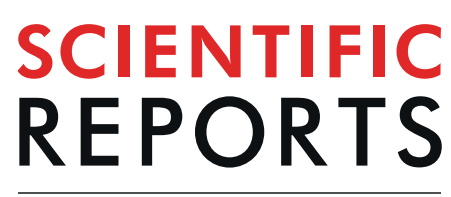

\title{
Assessment of Local Climate Zone Classification Maps of Cities in China and Feasible Refinements
}

\begin{abstract}
Chao Ren $\mathbb{1}^{1,2}$, Meng Cai ${ }^{3}$, Xinwei Li ${ }^{1 *}$, Lei Zhang ${ }^{1}$, Ran Wang ${ }^{3}$, Yong X $\mathrm{U}^{4}$ \& Edward $\mathrm{Ng}^{3}$
Local climate zone (LCZ) maps that describe the urban surface structure and cover with consistency and comparability across cities are gaining applications in studies of urban heat waves, sustainable urbanization and urban energy balance. Following the standard World Urban Database and Access Portal Tools (WUDAPT) method, we generated LCZ maps for over 20 individual cities and 3 major economic regions in China. Based on the confusion matrices constructed by manual comparison between the predicted classes and ground truths, we highlight the following: (1) notable variation in overall accuracies (i.e., 60\%-89\%) among cities were observed, which was mainly due to class incompleteness and distinct proportions of natural landscapes; (2) building classes in selected cities were poorly classified in general, with a mean accuracy of $48 \%$; (3) the sparsely built class (i.e., LCZ 9), which is rare in the selected Chinese cities, had the lowest classification accuracy ( $32 \%$ on average), and the class of low plants had the widest accuracy range. The findings indicate that the standard WUDAPT method alone is insufficient for generating LCZ products that demonstrate practical value, especially for built-up areas in China, and the misclassification is largely caused by the lack of building height data. This result is confirmed by a refinement test, in which the urban DEM retrieved from Sentinel-1 data with radar interferometry technique was used. The study shows a detailed and comprehensive assessment of applying the WUDAPT method in China and a feasible refinement strategy to improve the classification accuracy, especially for the built-up types of LCZ. The study could serve as a useful reference for generating quality-ensured LCZ maps. This study also examines and explores the relationship between socio-economic status and LCZ products, which is essential for further implementations.
\end{abstract}

Urbanization, as a process that gradually changes the physical landscape, is often accompanied by environmental problems and health challenges, such as urban heat islands, air quality degradation, heat stroke, overweight, hypertension, infection diseases and increasing deaths caused by injuries ${ }^{1-5}$. These challenges are being addressed with advances in urban climatology, where numerical and physical models in combination with urban canopy parameters (UCPs) have been used to study the interaction between city structure and local climate ${ }^{6-9}$. To acquire UCPs, the existing land use and land cover (LULC) data are usually compulsory. However, although several global LULC datasets are available ${ }^{10-12}$, they are inadequate for calculating UCPs because the description of urban morphology is deficient. To fill the data gap globally, the World Urban Database and Access Portal Tools (WUDAPT) project was initiated ${ }^{13,14}$. As a level 0 product, the local climate zone (LCZ) data have been developed to date. The LCZ scheme categorizes the urban surface into 10 built types and 7 natural types ${ }^{15,16}$, from which UCPs can be determined and thereby improved climate model results can be achieved. Due to its detailed description of urban areas, the LCZ scheme is gaining popularity in studies of local air/surface temperature features ${ }^{16-21}$, urban heat islands ${ }^{22-24}$, urban energy budgets ${ }^{25,26}$, outdoor thermal comfort ${ }^{27,28}$, machine learning ${ }^{20,29}$ and urban ventilation simulations ${ }^{30}$. When historical LCZ datasets are available, there are useful for examining the impacts of local cover change on the spatial pattern of land surface temperature ${ }^{31}$, evolution of the climatological effects of urbanization $^{32}$, and urban growth monitoring ${ }^{33}$.

Because the WUDAPT community provides a standard processing workflow for the generation of LCZ data from freely available satellite images (e.g., Landsat and Senitinel-2) and training samples that have been identified

${ }^{1}$ Faculty of Architecture, The University of Hong Kong, Hong Kong, China. ${ }^{2}$ Institute of Future Cities, The Chinese University of Hong Kong, Hong Kong, China. ${ }^{3}$ School of Architecture, The Chinese University of Hong Kong, Hong Kong, China. ${ }^{4}$ School of Geographical Sciences, Guangzhou University, Guangzhou, China. *email: lixinwei@hku.hk 


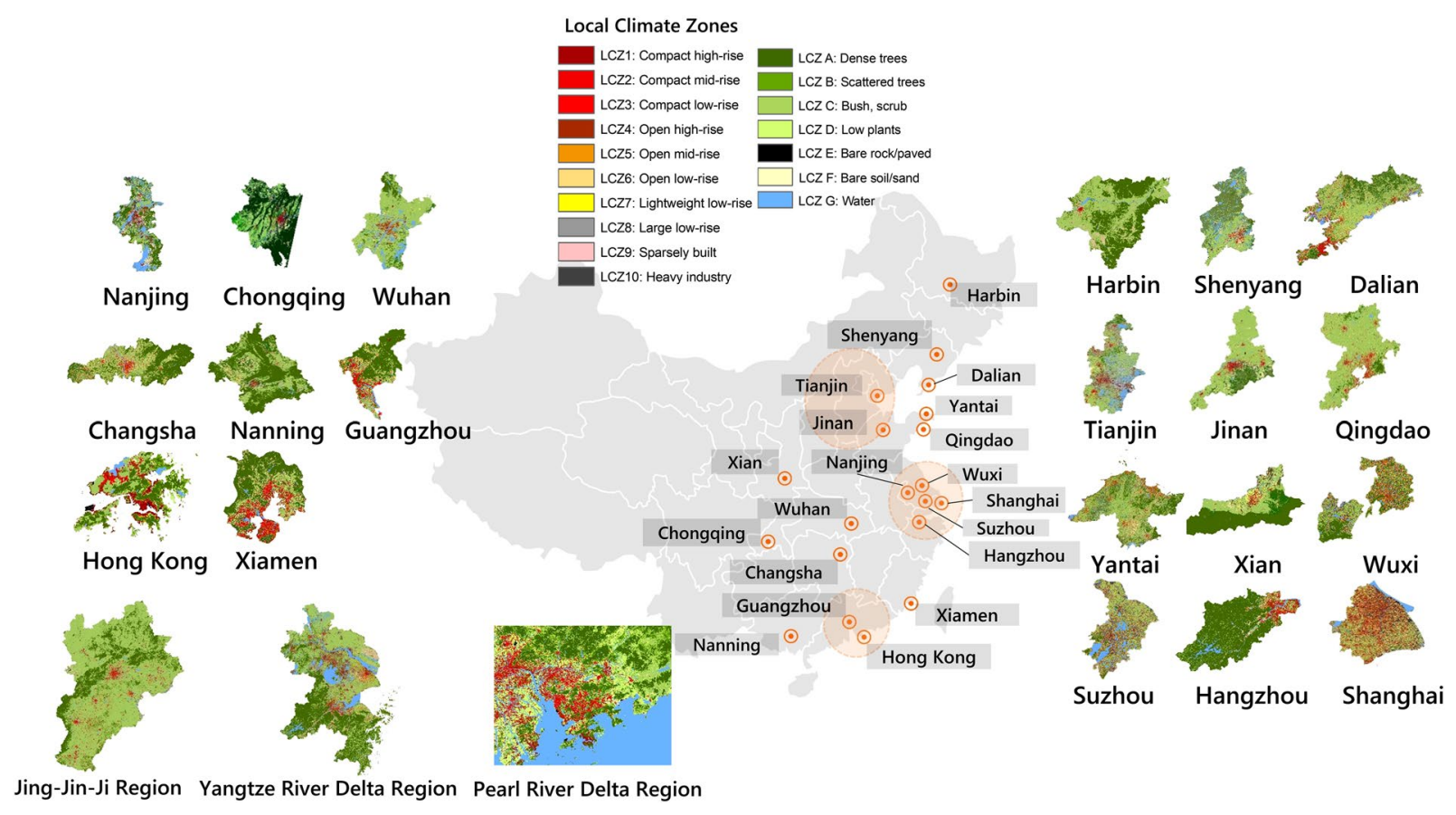

Figure 1. LCZ maps of 20 individual cities and 3 major economic regions.

according to Google Earth images with open source SAGA software, there is currently abundant LCZ data for more than 120 cities in different continents; these data are being produced and uploaded to the WUDAPT portal after quality assessments ${ }^{14,20}$. In this work, following the standard WUDAPT processing method, we generated LCZ maps for over 20 individual cities and 3 major economic regions in China from Landsat images acquired from 2014 to 2015. The cities vary in size and have distinct geographic locations; additionally, the cities have different levels of economic development. The quality assessment and cross-comparison among these LCZ maps are then rigorously conducted to reveal the factors that limit the accuracy of the WUDAPT method in Chinese cities. To explore the strategies that can improve the accuracy of current LCZ products, we evaluated the role of the urban digital elevation model (DEM) generated from Sentinel-1 data by the synthetic aperture radar interferometry (InSAR) technique. Although SAR data have been used for LCZ classification, only SAR intensity information has been adopted ${ }^{34-36}$, and the role of an InSAR-derived urban DEM for the refinement of LCZ products has not yet been investigated.

The contributions of the work are multi-fold. First, LCZ classification maps with a mean overall accuracy of $76 \%$ for more than 50 Chinese cities were generated, which enriched the database of land cover and land use in China and can benefit the study of urban climate ${ }^{24,37-39}$. Second, by mining the confusion matrices of such a large volume of LCZ data, the factors that limit the performance of the default WUDAPT workflow in cities of China have been revealed. Finally, this study sheds light on the directions of further optimization of the WUDAPT method by selecting suitable training samples, considering seasonal discrepancies between training samples and Landsat data, and involving external data sources. Among them, the urban DEM retrieved from freely available Sentinel-1 data shows great potential.

\section{Results}

Overall accuracies. Following the standard WUDAPT processing workflow ${ }^{40,41}$, the LCZ maps of 20 individual cities and 3 major economic regions (i.e., Jing-Jin-Ji, Yangzte River Delta, and Pearl River Delta) were produced (Fig. 1). As many as 15 out of the 20 selected cities can be found on the WUDAPT platform, while 5 cities and 3 economic regions are not yet submitted to the WUDAPT portal at this stage. The quality checking results and the availability of the LCZ map products used in this study are summarized in Table 1 . The overall accuracy of these LCZ classification maps ranges from $60 \%$ to $89 \%$, with a mean of $76 \%$ and a standard deviation of $7.2 \%$ (Fig. 2a). The overall accuracy has a similar fluctuation with the accuracy of natural land cover $\left(\mathrm{OA}_{\mathrm{n}}\right)$, which has a mean of $85 \%$ for the selected cities. Figure $2 \mathrm{a}$ also indicates a relatively poor and fluctuating performance of the classifier for built-up areas. The mean accuracy over built-up areas $\left(\mathrm{OA}_{\mathrm{u}}\right)$ is $47 \%$, and the lowest is only $26 \%$ in the Jing-Jin-Ji region. When compared to the number of training samples shown in Fig. 2(b), there is no statistically significant correlation with these accuracy measures.

Accuracy variation among classes. When examining the accuracy matrix of all classes in all cities/regions (Fig. 3a), it is observed that the class of LCZ 9 (sparsely built) has a consistently low accuracy, with a mean of $32 \%$ regardless of where the city is located and how developed the city is. It is followed by LCZ C (bush, scrub) and LCZ 5 (open mid-rise), with a mean of approximately $40 \%$. Over built-up areas, serval zero accuracies were observed, mainly appearing in LCZ 6 (open low-rise), LCZ 8 (large low-rise), LCZ 9, and LCZ 10 (heavy industry). On the other hand, three natural LCZ classes (i.e., LCZ A (dense trees), LCZ D (low plants) and LCZ G 


\begin{tabular}{|l|l|l|l|l|l|}
\hline WUDAPT Status & Cities & Wuxi \\
\hline In preparation & Nanjing & Hong Kong & Xiamen & Suzhou & Wuin \\
\hline Accept & Shenyang & & & & \\
\hline Minor & Wuhan & Changsha & Qingdao & Xian & Shanghai \\
\hline Major & Jinan & Tianjin & Hangzhou & & \\
\hline \multirow{2}{*}{ Not available } & Chongqing & Nanning & Harbin & Dalian & Yantai \\
\cline { 2 - 7 } & Guangzhou & & & & \\
\hline
\end{tabular}

Table 1. The availability and status of LCZ maps on the WUDAPT platform (in addition to "not available" cities, all other cities can be checked on the WUDAPT website; cities with "minor" or "accepted" status can be downloaded).

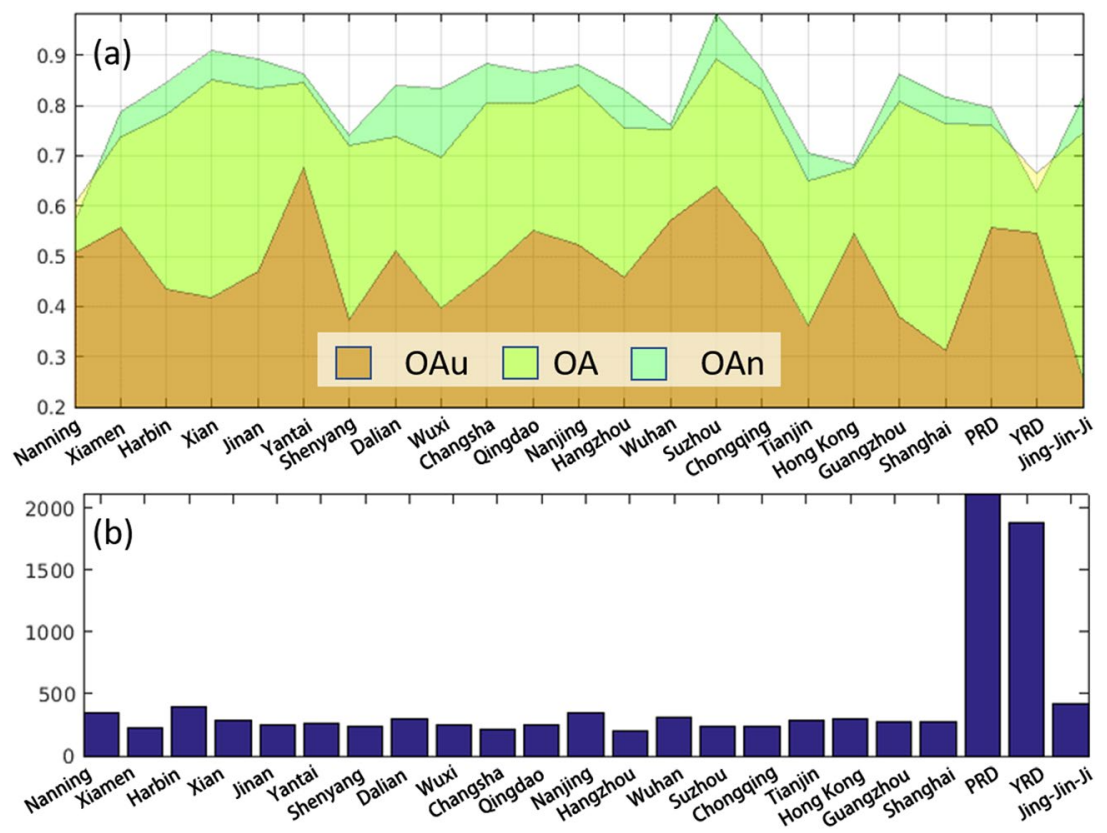

Figure 2. (a) Accuracy measures of LCZ maps for selected cities. (OAu: overall accuracy results of built-up classes; OA: overall accuracy results of all classification; OAn: overall accuracy results of natural land cover classes); (b) Total number of collected training samples for each study area.

(water)) were satisfactorily categorized in all cities, with a mean accuracy of $94 \%, 85 \%$ and $95 \%$, respectively. To reflect the contribution of cities to the classification accuracy and discrepancies among these classes, an area map is shown in Fig. 3b. It is observed that, for classes with high accuracy (e.g., LCZ A), the performance of the WUDAPT method in all cities is stable regardless of the variation in the number of training samples, while for the poorly classified LCZs (e.g., LCZ 9), the performance fluctuates notably, indicating that single-city optimization might be needed for the improvement of classifications with low accuracy.

Confusion matrix. By investigating the confusion matrix, the accuracy discrepancy could be explained by misclassification among the LCZ classes. To obtain a general picture of the class mixture over all cities, we accumulate the confusion matrices by summing the corresponding elements (Fig. 4a). It is clear that a large portion of the LCZ classes have misclassification errors. For example, the worst-classified LCZ 9 was mistakenly recognized as LCZ D in $56 \%$ of cities. As the land cover of heavy industry (LCZ 9) could be compact/open/large low-rise (LCZ 3, LCZ 6 and LCZ 8, respectively) and possibly contain large paved surfaces (LCZ E), confusion among these classes is common in the selected cities. To better understand how often these classes could be confused, the frequency of each class based on the confusion matrix was counted and the results are shown in Fig. $4 \mathrm{~b}$. It can be seen that classes in built areas (i.e., LCZs 1-10) are notably confused, and the pattern is distinct from the similarity matrix used for calculating the weighted accuracy ${ }^{42}$ and from those published ${ }^{35}$. As a comparison, Fig. 4c shows the combined confusion matrix from LCZ maps for Moscow, Warsaw, Yangon and Karachi, which is also distinct from that for Chinese cities. In addition to the urban classes, LCZ D (low plants), a class of natural surfaces, has the widest mixture range. It was mistakenly identified as LCZs 6, 9, B (scattered trees), C and F (bare soil or sand), all of which have a common feature of scattered trees according to their definitions.

Accuracy variation among cities. When focusing on the quality assessment in each city, it is noticed that approximately half of the cities do not contain all the LCZ classes, among which, the city of Suzhou has the fewest 

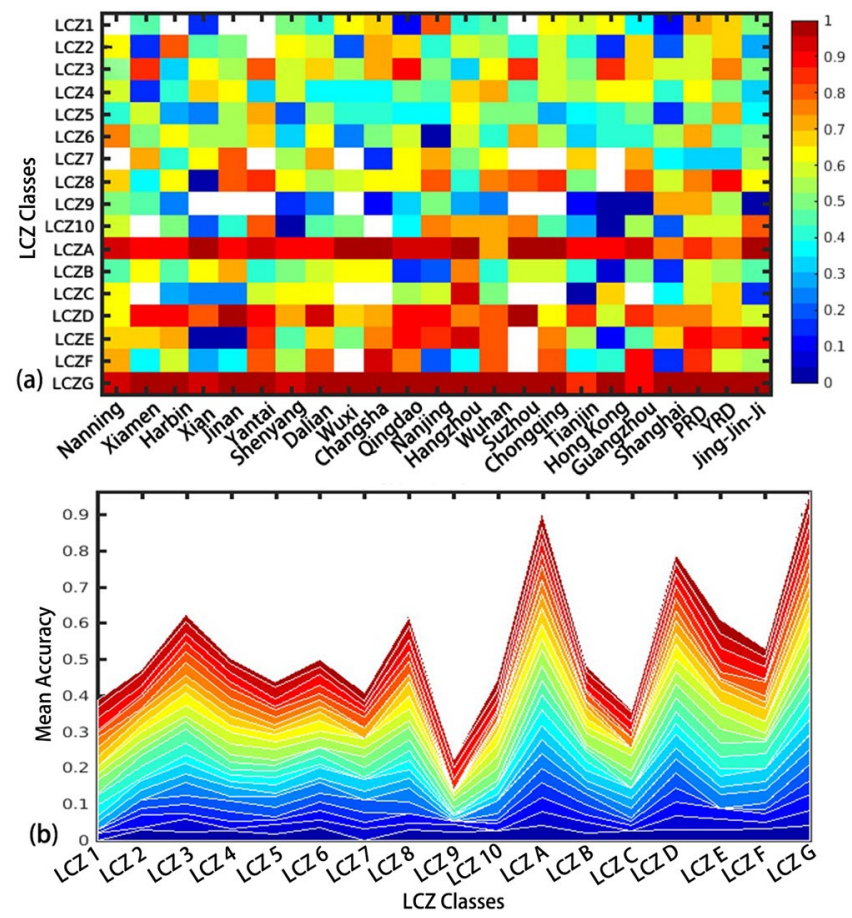

Figure 3. (a) The accuracy of each class in all selected cities/regions (X axis shows the name of the cities and regions; Y axis shows the $17 \mathrm{LCZ}$ classifications; the coloured bar shows the LCZ classification accuracy results ranging from $0-1)$; (b) the area map of (a) indicating the mean accuracy of each class and the contribution of each city/region (lines represent 20 different cities and 3 economic regions; $X$ axis shows the 17 LCZ classifications; $\mathrm{Y}$ axis shows the classification accuracy results ranging from $0-1$ ).

classes (6 less). Poor classifications in the selected cities mainly occur in built-up areas, with exceptions in that Xian, Jian and Tianjin have the worst classifications of natural lands. In these cities, zero accuracy was observed in LCZ E (bare rock or paved) and LCZ C (low plants), where there were misclassifications with LCZ F in Xian, LCZ 10 in Jinan and LCZ B in Tianjin. To investigate the relationship of classification accuracies among cities, following Eq. (2), we calculated the correlation matrix where the upper triangular part represents the correlation coefficients of urban classes and the lower part represents the coefficients of natural classes (Fig. 5). It is clear that the selected cities have comparable accuracies in each class of natural areas, while the correlation of accuracies of built-up classes among cities is generally low regardless of the LCZ type or city size. Considering that the performance of classifiers in natural lands is satisfied, it comes as no surprise that cities with less compact and open built-up classes (i.e., LCZs 1-6) usually have higher overall accuracies, e.g., Suzhou, Nanjing, Yantai, and Xian.

LCZ map refinement with urban DEM. The analysis of the confusion matrices indicates that the WUDAPT method is insufficient for identifying distinct building classes and that height information is expected to be vital for refinement. There are several techniques to acquire urban elevation data (e.g., LiDAR and photogrammetry); however, the cost is usually prohibitive, especially for large-scale mapping. To improve the accuracy of classifying urban built-up classes and staying in line with the philosophy of WUDAPT, that is, using freely available imagery, we generated the urban DEM with $25 \mathrm{~m}$ resolution from C-band Sentinel-1 SAR data using our self-developed TCPInSAR processor to detect the impact of adding then urban DEM into the LCZ scheme. Figure 6(a) shows the elevation map over an area in the PRD region. According to the definitions of the LCZ classes, we use thresholds of $10 \mathrm{~m}$ and $30 \mathrm{~m}$ to distinguish low-rise, midrise and high-rise classes. The classification map is shown in Fig. 6(b), whose resolution has been down-sampled to $100 \mathrm{~m}$. To make a cross-comparison, we extracted and shrank the LCZ building map into three classes by combining LCZ 1 and LCZ 4 as the high-rise class, LCZ 2 and LCZ 5 as the midrise, and others as the low-rise, as shown in Fig. 6(c). Figure 6(d) shows the corresponding map constructed from the InSAR-derived urban DEM. Significant discrepancies are observed between Fig. 6(c,d), which indicates the uncertainties associated with the current LCZ products. These discrepancies also showed the feasibility of using InSAR-derived DEM products in the refinement of classifying LCZ urban classes.

Correlation among cities in class distribution. Although the current accuracy of urban classes might not be sufficient to support a comprehensive analysis of the spatial pattern of each single class, it is possible to reveal the similarity among cities and regions with respect to class distribution by correlation analysis. Following Eq. (2), we calculated the correlation coefficient of the proportion of LCZ urban classes among the selected cities, as shown in Fig. 7. In general, the urban structures of cities have a low similarity among all selected cities regardless of city size, geographic location, and social economic status (see Fig. 7(a)), which indicates that unique 

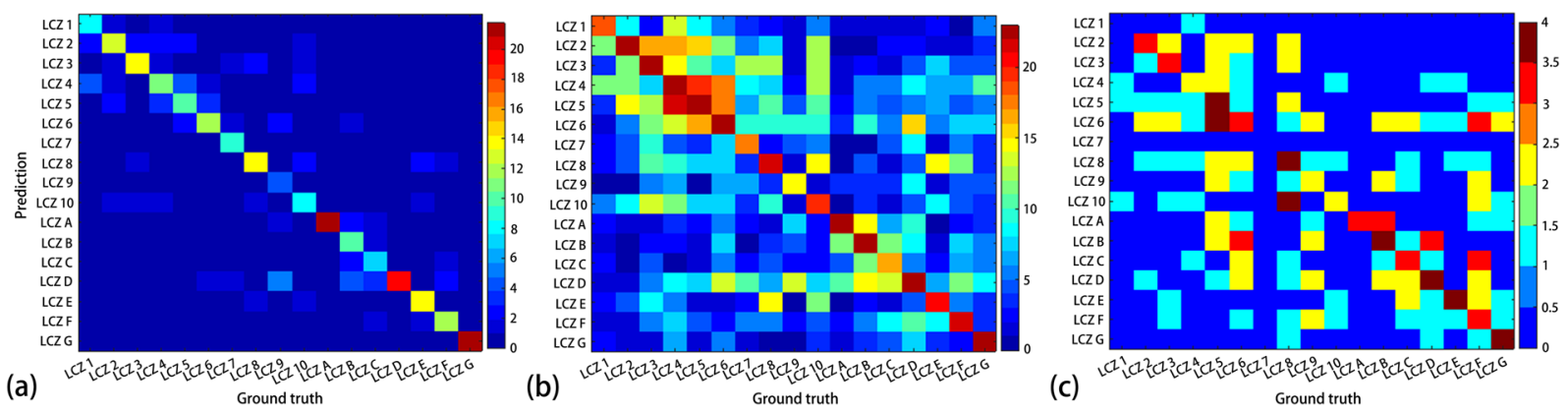

Figure 4. (a) Accumulation of normalized confusion matrix where the original elements have been divided by the row total; (b) appearance number of each class in all the confusion matrices; (c) appearance number of each class in the confusion matrices of Moscow, Warsaw, Yangon and Karachi. The coloured bar shows the number of cities.

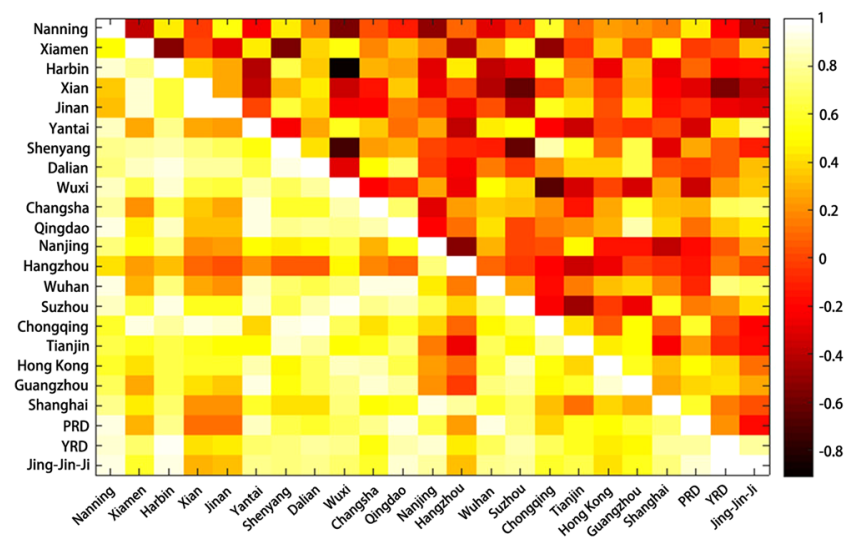

Figure 5. The correlation matrix of classification accuracies among cities/regions. The upper triangular part shows the correlation coefficients of urban areas, and the lower triangular part shows those of natural lands. The coloured bar shows the correlation coefficients ranging from -1 to 1 .

urban canopy parameters should be determined for each city when applying numerical models to simulate the weather, climate and air quality. However, when looking into the cities within each economic region, the correlation appears to be notable. Cities located in the same economic region tend to have similar proportions of urban LCZ classes. In the Yangtze River Delta region, except for the city of Nantong, the other 13 cities have a high correlation $(>0.8)$ in class proportion with one or more cities (Fig. 7(b)). A higher correlation $(>0.9)$ among most of the cities was also observed in the Jing-Jin-Ji region, where only the cities of Chengde and Zhangjiakou were distinct from the other 11 cities (Fig. 7(c)). The correlation matrix over cities in the Pearl River Delta region is shown in Fig. $7(\mathrm{~d})$, where the class distributions in cities maintain a correlation of at least 0.8 except for that in Hong Kong, which, as expected, as one of the most developed cities in the world, was different from the other cities in the region.

\section{Discussion}

The generated LCZ maps have notable variations in classification accuracies (especially for the built-up classes). We attribute this result to the following reasons: (1) class incompleteness. It is commonly accepted that the WUDAPT method has relatively poor classification performance for compact and open buildings (i.e., LCZs 1-6). Therefore, for cities that lack such classes, a higher classification quality can be anticipated. Examples in this work are Yantai and Suzhou. (2) Insufficient training samples. Although the significant relationship between the number of training samples and accuracy was not observed in the selected cities, we noticed that Hong Kong, owning the highest sample density in built-up areas, has the best urban classification performance, while the worst is the Jing-Jin-Ji region, where the sample density is lowest. Using LCZ 9 as another example, most cities performed poorly in this class except for Harbin, where the training samples were rather abundant, up to $28 \%$ of the total amount. (3) Similarity among classes. From the generated LCZ maps, it is found that, in addition to the misclassification among compact and open buildings, a large portion of classes were mistakenly recognized as LCZ 10 and LCZ D in the selected cities. Indeed, industrial structures could contain compact or open buildings. (4) Seasonal mismatch. When selecting training samples from Google Earth, seasonal differences with Landsat images deserve considerable attention. It is particularly risky for cities with four distinct seasons. Figure 8 shows the land features of a training sample collected in the Jing-Jin-Ji region in different months, indicating that a seasonal mismatch could exist between the training samples and the Landsat and might cause misclassification. 


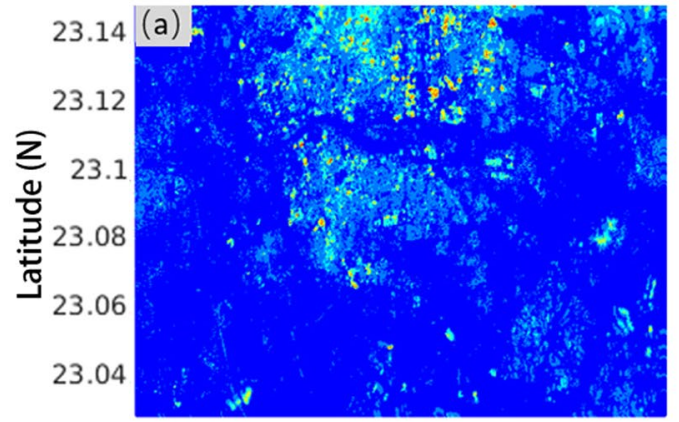

$113.2 \quad 113.25 \quad 113.3 \quad 113.35113 .4$

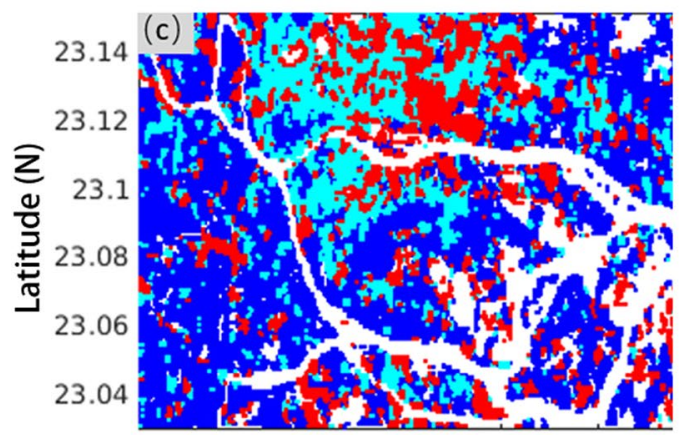

113.2113 .25113 .3113 .35113 .4 Longitude (E)

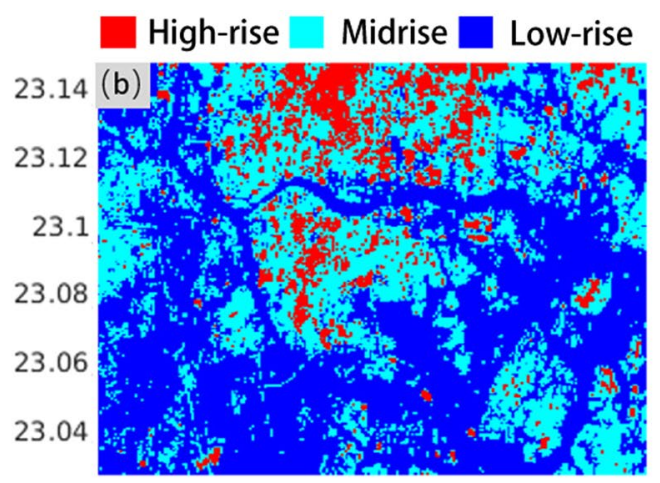

113.2113 .25113 .3113 .35113 .4

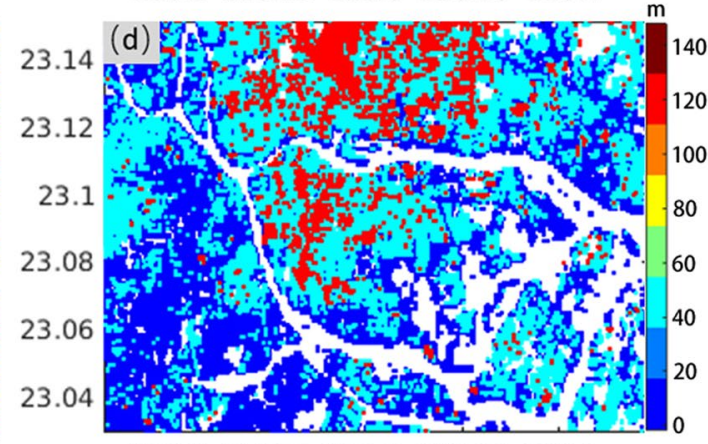

$113.2113 .25113 .3 \quad 113.35113 .4$ Longitude $(\mathrm{E})$

Figure 6. (a) Urban DEM retrieved from Sentinel-1 data; (b) classifications according to mean heights over $100 \mathrm{~m}$ patches; (c) height-related classifications extracted from the LCZ map; (d) height-related classifications extracted from $(\mathbf{b})$.

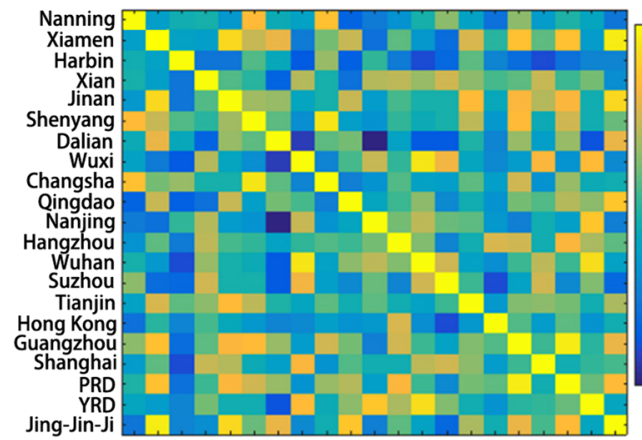

(a)
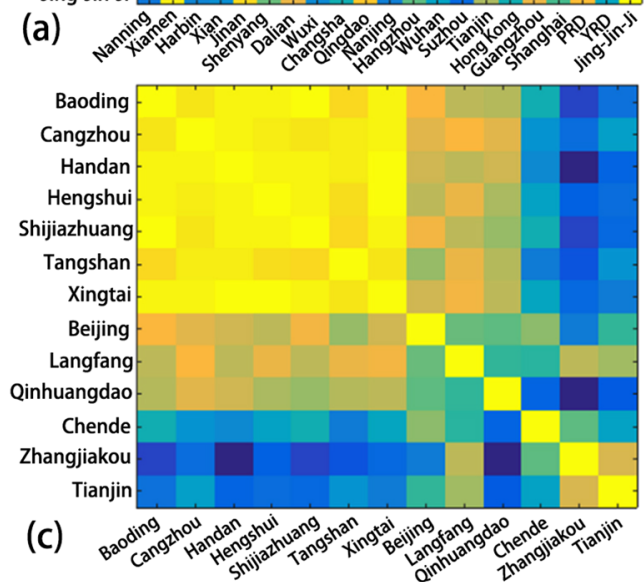

Figure 7. Correlation in class proportions among (a) the selected cities/regions; (b) cities in the Yangtze River Delta region; (c) cities in the Jing-Jin-Ji region; and (d) cities in the Pearl River Delta region. The coloured bar shows the correlation coefficients ranging from -1 to 1 .

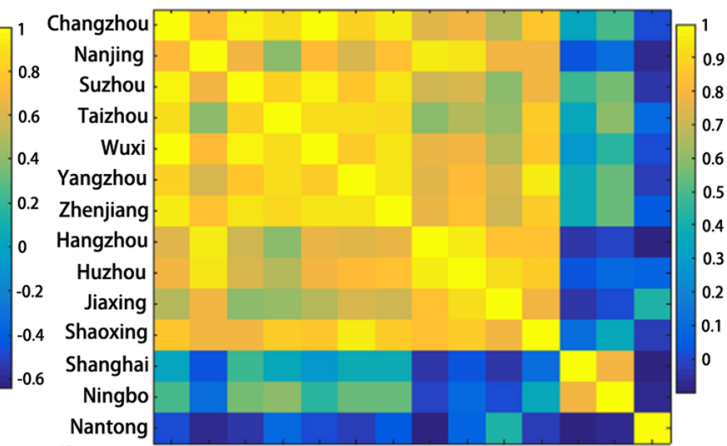

(b)

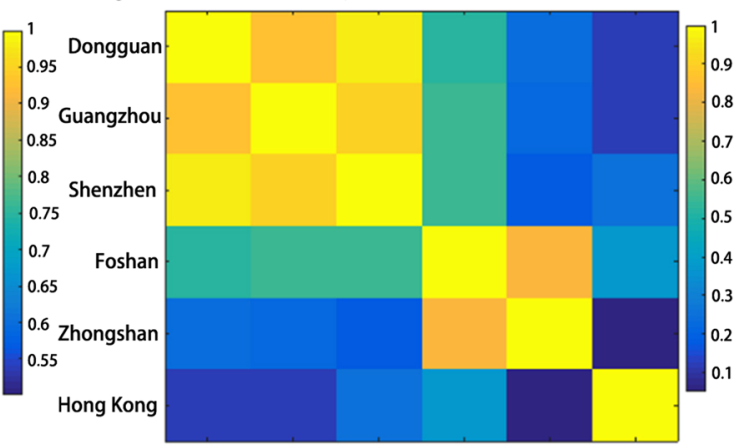

(d)

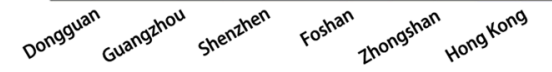



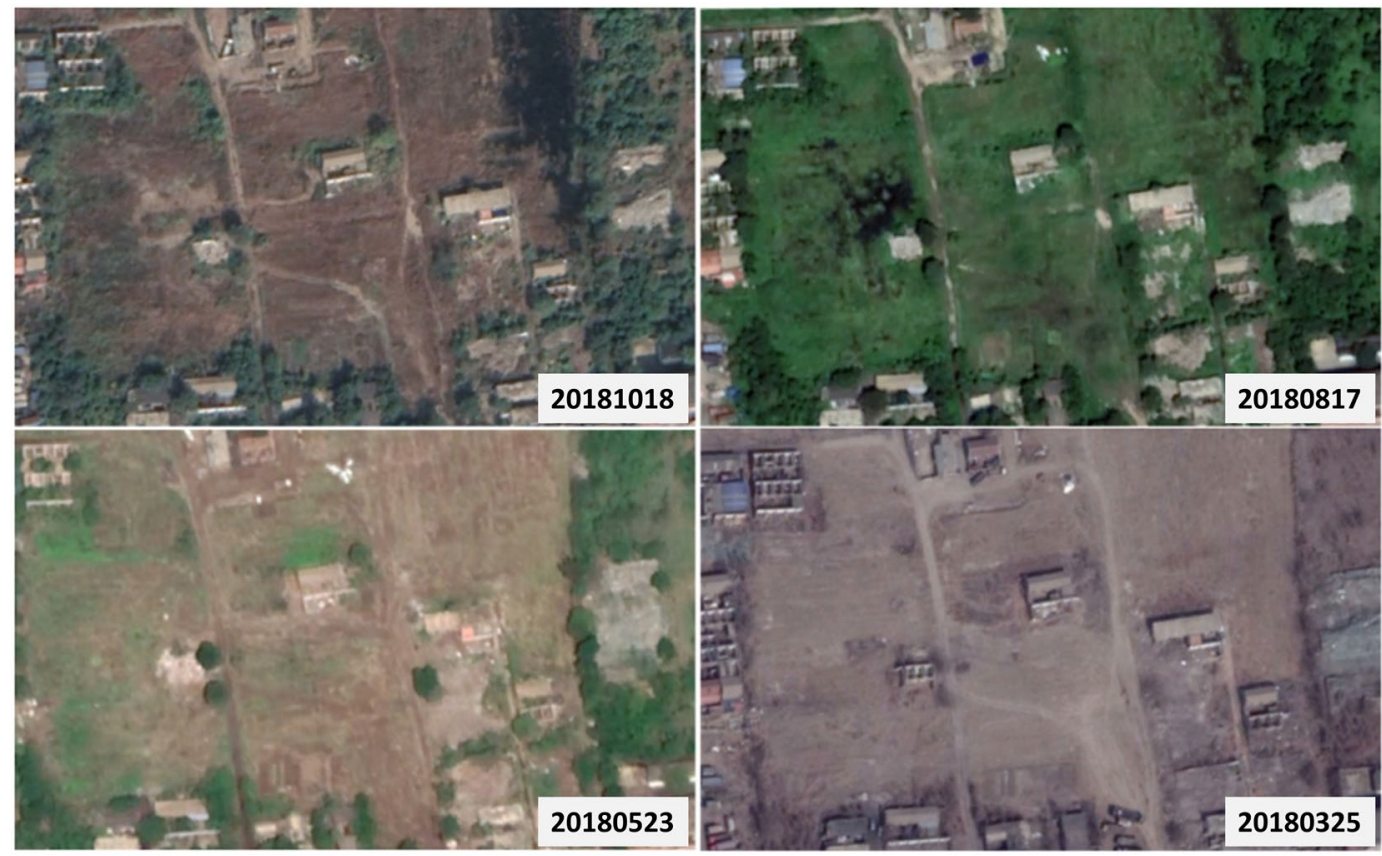

Figure 8. Spatial features of the same training sample in different seasons. The figures were captured from: Google, DigitalGlobe.

During the generation of LCZ maps, to improve the efficiency, we jointly processed some cities as a cluster. Among the three major economic regions, the Yangtze River Delta and Pearl River Delta have comparable (or even higher) classification accuracies, while the performance (especially in built-up areas) over Jing-Jin-Ji is relatively poor, mainly due to insufficient samples (only 420 over the whole territory). Such practice confirms that large-scale processing is feasible provided that cities share similar biophysical backgrounds and the features of training samples are consistent.

When looking into the confusion matrices, severe misclassification mainly occurred among urban classes in the selected cities. To distinguish them, information about the relative locations and heights of buildings is vital; however, optical images are insufficient to identify building heights, likely leading to the incorrect selection of training samples and thereby incorrect classifications. Building heights seem to be compulsory for accurate classification of urban landscapes, which was verified by a preliminary experiment with InSAR-derived urban DEM. Although the urban DEM has a moderate resolution of $25 \mathrm{~m}$, it provides more reasonable height-related classifications, indicating that building classifications can be refined with extra height information.

The correlation analysis among cities reveals that similarity in class proportions is regionally dependent. Cities within the same economic region have rather high correlation coefficients, indicating they have a higher similarity in the proportions of different built-up types, while the correlations among all cities are relatively low. The potential causes include similar social, economic and climate status. However, within each region, there are still cities with distinct urban structures, and this situation leads to low correlations. The distinction is mainly raised by one or more classes that have extraordinary proportions. For example, Nantong in the Yangtze River Delta region has $48.1 \%$ of sparsely built (i.e., LCZ 9) land covers in urban areas, which is much higher than that observed in other cities. Hong Kong in the Pearl River Delta region is distinct from the others by having $30.6 \%$ of the compact high-rise class (i.e., LCZ 1), the highest in the region. The high similarity in LCZ classes among cities within respective regions might indicate that the convergence of city development is serious, which can serve as a reference for regional planning and policy making.

\section{Conclusion}

Following the standard WUDPT processing workflow, the LCZ maps were generated for 20 individual cities and 3 major economic regions in China based on site-specific operation. By examining the difference in classification accuracy, we conclude that the accuracy discrepancy among cities is mainly due to class incompleteness (especially the compact classes), the number and quality of training samples, the similarity among classes and the seasonal mismatches between samples and Landsat data. This result indicates that because Google Earth's image does not include the height information of buildings, the standard WUDAPT method is inadequate for distinguishing height-related classes. Considering that the similarity among classes often makes the sample collection rather challenging, even for experienced operators, novel classifiers other than supervised ones should be developed. For cities with distinct seasonal land features (especially those in northern China), when collecting training samples, seasonal consistency should be ensured, as low plants (LCZ D) in summer could become bare soil (LCZ F) in winter in these cities. 
The experiment with the urban DEM demonstrated a reasonable refinement, which shows that the height information retrieved from satellite SAR images (e.g., Sentinel-1) has the potential for distinguishing and detecting the building heights of LCZ classes 1-10 in built-up areas and can improve the current WUDAPT method and level 0 product accuracy. However, although Sentienl- 1 data are freely available around the world, it is challenging for non-InSAR experts to extract the urban DEM from these data. The main difficulty lies in the mitigation of building/ground deformations and turbulent atmospheric delays, which are mixed with building heights in InSAR measurements. Moreover, how to tightly integrate the data in the WUDAPT method (e.g., dataset alignment and height classification threshold selection) also needs further investigation. To facilitate the usage of the urban DEM in LCZ classification, the generation of annually updated national urban elevation datasets from interferometric Sentinel-1 measurements will be a part of our future work, which is also expected to benefit WUDAPT L1 (i.e., 2.5D urban forms) data production.

It is worth noting that, although the building classes are currently of moderate accuracy, i.e., approximately $40 \%$, the potential of LCZ maps can also be explored as they carry more detailed information on urban morphology than do other classification datasets and they are valued at both the domestic and the international levels. At the national level, the development of LCZ maps for 20 individual cities and 3 major economic regions in China can serve as a fundamental database for various applications, such as urban climate investigation ${ }^{32}$, urban energy consumption estimation ${ }^{26}$, urbanization projection and air pollution distribution detection ${ }^{43}$. The correlation analysis results of the developed LCZ data show the morphological characteristics of both built-up areas and natural landscapes of Chinese cities and regions. The convergence of city development within respective economic regions might be useful for evaluating the collaborative/competitive relationship and degree of regional integration. At the international level, the developed database is also a crucial part of the WUDAPT database because approximately half of the training samples and LCZ maps are from this study. The developed database and training samples not only lead to the development of global and regional LCZ maps but also make data fusion and data transferability possible. For example, our data were adopted in the latest global transferability of local climate zone models ${ }^{44}$. Based on our findings, the study also indicates the use of open data to develop an urban DEM for improving the LCZ classification accuracy of built-up areas.

Moreover, when studying the meteorological effects of urbanization, the LCZ maps can be reduced to 3 classes (i.e., urban low-rise, midrise and high-rise) as one of the inputs of the Weather Research and Forecasting (WRF) model to enhance the simulation ${ }^{32}$. Meanwhile, the active WUDAPT community is nurturing innovative methods to produce high-quality LCZ products, e.g., by involving other types of data sources and using advanced machine learning techniques. Accurate and publicly accessible LCZ maps for Chinese cities can be expected in the near future.

\section{Dataset and Methodology}

Cities. A total of 20 individual cities and 3 major economic regions (i.e., Jing-Jin-Ji, Yangzte River Delta, and Pearl River Delta) were selected for LCZ map generation (Fig. 1). Although a large portion of cities are in the eastern segment of the country, they are representative, as the selected cities range from the first (i.e., megacities) to the second (i.e., large-medium cities) to the third-tier levels (i.e., medium cities). These cities contributed more than $50 \%$ to China's GDP in 2018. Considering the ecoregion similarity ${ }^{20}$, the cities in China's three major regions are jointly processed.

Data. The $30 \mathrm{~m}$ Landsat 8 level 1 images from 2014 to 2015 were downloaded from the U.S. Geological Survey (http://glovis.usgs.gov). They were used for LCZ map generation for the cities in China. Seamless mosaic and atmospheric correction were conducted if necessary, and all images were down-sampled to $100 \mathrm{~m}$ to reflect the local urban structures instead of single objects. For urban DEM generation, a set of Sentinel-1 radar data acquired in 2015 with a spatial resolution of $25 \mathrm{~m}$ were collected from the European Space Agency (https://scihub.copernicus.eu/dhus/\#/home).

Training samples. Training data are vital for LCZ classification in the standard WUDAPT workflow. In view of efficiency, it is ideal to classify a large number of cities by using the training data collected in one or a few cities. However, such a strategy usually fails to produce satisfied classification data due to transferability, a common issue faced by machine learning methods, which has also been recently reported ${ }^{20}$. Therefore, in our work, although it is time consuming, we generated the training polygons for each LCZ class in every city/region following the guidance on the WUDAPT website. To distinguish the classes that appear similar in Google Earth images (e.g., compact low-rise (LCZ3), open low-rise (LCZ6) and lightweight low-rise (LCZ7)), Google Street View images were also employed. Considering that the Landsat images were acquired during a two-year period, when selecting training samples, we avoided digitizing polygons in places that were likely to change, such as construction sites, vacant development areas and bare land ${ }^{19}$. We selected a total of 9923 samples from these cities. The number of samples for each city/region is shown in Fig. 2b. It is worth noting that some types of LCZ classes were not available in these cities. To reduce the effect of sample imbalances, we selected a similar number of samples from each class, provided that it was abundant in the cities.

WUDAPT processing workflow. There are 3 steps involved in the standard WUDAPT processing workflow for the generation of LCZ maps ${ }^{13-15}$ : (1) acquisition and pre-processing of freely available satellite images (e.g., Landsat-8); (2) selection of training samples by experienced operators for each city; (3) conduction of supervised classification (e.g., random forest) embedded as an 'LCZ classification tool' in SAGA GIS. The workflow can be efficiently applied in each city while considering that a large portion of selected cities are located in the top 3 megacity clusters of China and that they share similar biophysical backgrounds; based on this information, we combined the samples in each cluster and generated the entire LCZ map for the region ${ }^{45}$. 
Quality assessment and correlation coefficients. To evaluate the accuracy of the LCZ maps, a random sampling scheme (i.e., bootstrapping) is used in the WUDAPT workflow ${ }^{14}$, where the training samples are divided into two portions for training and evaluation. Considering that the collection of a sufficient number of samples requires a long period and that only a limited number of samples can be selected for the classes that are rare in a given city, we conduct the quality assessment manually after the generation of LCZ maps using all samples. A new set of samples was randomly collected for each LCZ class as ground truths. The number of ground truths was set to $0.5 \%$ of each generated class. By cross-comparison between the ground truths and produced LCZ maps, the confusion matrices can then be determined, and these matrices reflect the misclassification of land covers of each city. To reflect the overall misclassification of all the selected cities/regions, an accumulated confusion matrix was also determined, where the number of evaluation samples was replaced by the percentage to address the ground truth imbalance among cities. Based on the single-city confusion matrix, a set of quality metrics can be calculated. We employed the following accuracies to evaluate the quality of the LCZ maps ${ }^{14,20}$ : (1) overall accuracy $(\mathrm{OA})$, which denotes the percentage of correctly classified pixels, regardless of the performance of each class; (2) $\mathrm{OA}_{b}$ reflects the overall accuracy of the LCZ classes related only to built-up areas; and (3) $\mathrm{OA}_{\mathrm{n}}$ represents the overall accuracy of the LCZ classes of natural land covers. They can be calculated as follows:

$$
O A=\frac{\sum_{i=1}^{17} N_{i}^{c}}{N^{a}} ; O A_{u}=\frac{\sum_{i=1}^{17} N_{i}^{c b}}{N_{\text {built }}^{a}}, O A_{n}=\frac{\sum_{i=1}^{17} N_{i}^{c n}}{N_{\text {natural }}^{a}}
$$

where $N_{i}^{c}, N_{i}^{c b}$ and $N_{i}^{c n}$ are the correctly classified areas of all classes, built-up classes and natural classes, respectively, and $N^{a} N_{b u i l t}^{a}$ and $N_{\text {natrual }}^{a}$ are the corresponding total ground truths. In addition to the aforementioned accuracy measures, there are two other commonly used metrics, i.e., $\mathrm{OA}_{\mathrm{bu}}$, which denotes the overall accuracy of the built versus natural LCZ classes, and the weighted accuracy $\left(\mathrm{OA}_{\mathrm{w}}\right)$, which is obtained by applying weights to the confusion matrix to account for the similarity and dissimilarity among classes. Considering that these two measures are usually high and could not reflect the discrepancies among cities, we discarded them in this work. To reflect the reliability of the generated LCZ maps (i.e., how likely that the class on the map will actually be present on the ground), we also calculated the user's accuracy by taking the total number of correct classifications for a particular class and dividing it by the row total. Because a large number of LCZ maps were generated, we attempted to investigate the relationship among the cities by calculating the correlation coefficients that are defined as follows ${ }^{46}$.

$$
r_{x y}=\frac{\sum_{j=1}^{n}\left(x_{j}-\bar{x}\right)\left(y_{j}-\bar{y}\right)}{\sqrt{\sum_{j=1}^{n}\left(x_{j}-\bar{x}\right)^{2}} \sqrt{\sum_{j=1}^{n}\left(y_{j}-\bar{y}\right)^{2}}}
$$

where $\mathrm{n}$ is the number of LCZ classes (i.e., 17); $x_{j}$ and $y_{j}$ are the class accuracies or distributions in two cities, respectively.

Urban DEM generation. The synthetic aperture radar interferometry (InSAR) technique has long been used for DEM generation. Global SRTM DEM ${ }^{47}$ and TanDEM-X DEM ${ }^{48}$ are typical examples. To retrieve the urban DEM from Sentinel-1 data acquired with a repeating cycle of 12 days, it is compulsory to mitigate the contribution of ground/building deformation and atmospheric artefacts in InSAR measurements. To this end, we adopted our self-developed multi-temporal InSAR technique termed TCPInSAR ${ }^{49}$ to process the data. The estimator has several advanced features, e.g., robust image coregistration ${ }^{50}$, adaptive coherent point selection ${ }^{44}$, quad-tree model for atmospheric delay mitigation ${ }^{51}$ and parameter estimation with no need for phase unwrapping $^{52}$, which guarantees the quality of the retrieved DEM.

\section{Data availability}

The Landsat data are publicly available here: http://glovis.usgs.gov.The Sentinel-1 data are also publicly available from https://scihub.copernicus.eu/dhus/\#/home. For the WUDAPT product, only those after the quality checking are publicly available from: http://www.wudapt.org/.

Received: 12 June 2019; Accepted: 28 November 2019;

Published online: 11 December 2019

\section{References}

1. Gong, P. et al. Urbanisation and health in China. The Lancet 379, 843-852 (2012).

2. Moore, M., Gould, P. \& Keary, B. S. Global urbanization and impact on health. International Journal of Hygiene and Environmental Health 206, 269-278 (2003).

3. Van de Poel, E., O’Donnell, O. \& Van Doorslaer, E. Urbanization and the spread of diseases of affluence in China. Economics \& Human Biology 7, 200-216 (2009).

4. Patz, J. A., Campbell-Lendrum, D., Holloway, T. \& Foley, J. A. Impact of regional climate change on human health. Nature 438, 310-317 (2005).

5. Zhou, D., Zhao, S., Liu, S., Zhang, L. \& Zhu, C. Surface urban heat island in China's 32 major cities: spatial patterns and drivers. Remote Sensing of Environment 152, 51-61 (2014).

6. Ching, J. et al. WUDAPT: an urban weather, climate, and environmental modeling infrastructure for the anthropocene. Bulletin of the American Meteorological Society 99, 1907-1924 (2018).

7. Ching, J. A perspective on urban canopy layer modeling for weather, climate and air quality applications. Urban Climate 3, 13-39 (2013).

8. Masson, V. Urban surface modeling and the meso-scale impact of cities. Theoretical applied climatology 84, 35-45 (2006). 
9. Hammerberg, K., Brousse, O., Martilli, A. \& Mahdavi, A. Implications of employing detailed urban canopy parameters for mesoscale climate modelling: a comparison between WUDAPT and GIS databases over Vienna, Austria. International Journal of Climatology 38, e1241-e1257 (2018).

10. Chen, J. et al. Global land cover mapping at 30m resolution: A POK-based operational approach. ISPRS Journal of Photogrammetry and Remote Sensing 103, 7-27 (2015).

11. Bartholomé, E. \& Belward, A. S. GLC2000: a new approach to global land cover mapping from earth observation data. International Journal of Remote Sensing 26, 1959-1977 (2005).

12. Friedl, M. A. et al. Global land cover mapping from MODIS: algorithms and early results. Remote Sensing of Environment 83, 287-302 (2002).

13. Bechtel, B. et al. Mapping local climate zones for a worldwide database of the form and function of cities. ISPRS International Journal of Geo-Information 4, 199-219 (2015).

14. Bechtel, B. et al. Generating WUDAPT level 0 data - current status of production and evaluation. Urban Climate 27, 24-45 (2019).

15. Bechtel, B. \& Daneke, C. Classification of local climate zones based on multiple earth observation data. IEEE Journal of Selected Topics in Applied Earth Observations and Remote Sensing 5, 1191-1202 (2012).

16. Stewart, I. D. \& Oke, T. R. Local climate zones for urban temperature studies. Bulletin of the American Meteorological Society 93, $1879-1900$ (2012).

17. Geletič, J., Lehnert, M. \& Dobrovolný, P. Land surface temperature differences within local climate zones, based on two central European cities. Remote Sensing 8, 788 (2016).

18. Fenner, D., Meier, F., Bechtel, B., Otto, M. \& Scherer, D. Intra and inter local climate zone variability of air temperature as observed by crowdsourced citizen weather stations in Berlin, Germany. Meteorologische Zeitschrift 26, 525-547 (2017).

19. Cai, M., Ren, C., Xu, Y., Lau, K. K.-L. \& Wang, R. Investigating the relationship between local climate zone and land surface temperature using an improved WUDAPT methodology - a case study of Yangtze River Delta, China. Urban Climate 24, 485-502 (2018).

20. Demuzere, M., Bechtel, B. \& Mills, G. Global transferability of local climate zone models. Urban Climate 27, 46-63 (2019).

21. Quanz, A. J., Ulrich, S., Fenner, D., Holtmann, A. \& Eimermacher, J. Micro-Scale Variability of air temperature within a local climate zone in Berlin, Germany, during summer. Climate 6, 5 (2018)

22. Leconte, F., Bouyer, J., Claverie, R. \& Pétrissans, M. Using local climate zone scheme for UHI assessment: evaluation of the method using mobile measurements. Building and Environment 83, 39-49 (2015).

23. Emmanuel, R. \& Krüger, E. Urban heat island and its impact on climate change resilience in a shrinking city: the case of Glasgow, UK. Building and Environment 53, 137-149 (2012).

24. Kotharkar, R. \& Bagade, A. Evaluating urban heat island in the critical local climate zones of an Indian city. Landscape and Urban Planning 169, 92-104 (2018).

25. Alexander, P. J., Mills, G. \& Fealy, R. Using LCZ data to run an urban energy balance model. Urban Climate 13, 14-37 (2015).

26. Quan, S. J., Dutt, F., Woodworth, E., Yamagata, Y. \& Yang, P. P.-J. Local climate zone mapping for energy resilience: a fine-grained and 3D approach. Energy Procedia 105, 3777-3783 (2017).

27. Lau, K. K.-L., Chung, S. C. \& Ren, C. Outdoor thermal comfort in different urban settings of sub-tropical high-density cities: an approach of adopting local climate zone (LCZ) classification. Building and Environment 154, 227-238 (2019).

28. Lamarca, C., Qüense, J. \& Henríquez, C. Thermal comfort and urban canyons morphology in coastal temperate climate, Concepción, Chile. Urban Climate 23, 159-172 (2018).

29. Xu, G., Zhu, X., Tapper, N. \& Bechtel, B. Urban climate zone classification using convolutional neural network and ground-level images. Progress in Physical Geography: Earth and Environment 43, 410-424 (2019).

30. Yang, J. et al. Local climate zone ventilation and urban land surface temperatures: towards a performance-based and wind-sensitive planning proposal in megacities. Sustainable Cities and Society 47, 101487 (2019).

31. Wang, R. et al. Detecting multi-temporal land cover change and land surface temperature in Pearl River Delta by adopting local climate zone. Urban Climate 28, 100455 (2019).

32. Tse, J. W. P. et al. Investigation of the meteorological effects of urbanization in recent decades: a case study of major cities in Pearl River Delta. Urban Climate 26, 174-187 (2018).

33. Danylo, O., See, L., Gomez, A., Schnabel, G. \& Fritz, S. In EGU General Assembly Conference Abstracts. 18043

34. Bechtel, B., See, L., Mills, G. \& Foley, M. Classification of local climate zones using SAR and multispectral data in an arid environment. IEEE Journal of Selected Topics in Applied Earth Observations and Remote Sensing 9, 3097-3105 (2016).

35. Qiu, C., Schmitt, M., Mou, L., Ghamisi, P. \& Zhu, X. X. Feature importance analysis for local climate zone classification using a residual convolutional neural network with multi-source datasets. Remote Sensing 10,1572 (2018).

36. Hu, J., Ghamisi, P. \& Zhu, X. X. Feature extraction and selection of sentinel-1 dual-pol data for global-scale local climate zone classification. ISPRS International Journal of Geo-Information 7, 379 (2018).

37. Cao, C. et al. Urban heat islands in China enhanced by haze pollution. Nature Communications 7, 12509 (2016)

38. Lang, W., Long, Y. \& Chen, T. Rediscovering Chinese cities through the lens of land-use patterns. Land Use Policy 79, 362-374 (2018).

39. Kuang, W., Liu, J., Dong, J., Chi, W. \& Zhang, C. The rapid and massive urban and industrial land expansions in China between 1990 and 2010: a CLUD-based analysis of their trajectories, patterns, and drivers. Landscape and Urban Planning 145, 21-33 (2016).

40. Bechtel, B. et al. CENSUS of cities: LCZ classification of cities (level 0)-workflow and initial results from various cities. Proceedings of the 9th International Conference on Urban Climate (Toulouse, France, 2015).

41. Mills, G., Ching, J., See, L., Bechtel, B. \& Foley, M. An introduction to the WUDAPT project. Proceedings of the 9th International Conference on Urban Climate (Toulouse, France, 2015).

42. Bechtel, B. et al. Quality of crowdsourced data on urban morphology-the human influence experiment (HUMINEX). Urban. Science 1, 15 (2017)

43. Shi, Y., Ren, C., Lau, K. K.-L. \& Ng, E. Investigating the influence of urban land use and landscape pattern on PM2.5 spatial variation using mobile monitoring and WUDAPT. Landscape and Urban Planning 189, 15-26 (2019).

44. Zhang, L., Ding, X. \& Lu, Z. Ground settlement monitoring based on temporarily coherent points between two SAR acquisitions. ISPRS Journal of Photogrammetry and Remote Sensing 66, 146-152 (2011).

45. Cai, M., Ren, C., Xu, Y., Dai, W. \& Wang, X. M. Local climate zone study for sustainable megacities development by using improved WUDAPT methodology - a case study in Guangzhou. Procedia Environmental Sciences 36, 82-89 (2016).

46. Kendall, M. \& Stuart, A. The advanced theory of statistics. Vol. 1: Distribution theory 4th edn (Griffin, 1977).

47. Jarvis, A., Reuter, H. I., Nelson, A. \& Guevara, E. Hole-filled SRTM for the globe Version 4, available from the CGIAR-CSI SRTM $90 \mathrm{~m}$ Database, http://srtm.csi.cgiar.org 2008).

48. Rossi, C., Rodriguez Gonzalez, F., Fritz, T., Yague-Martinez, N. \& Eineder, M. TanDEM-X calibrated raw DEM generation. ISPRS Journal of Photogrammetry and Remote Sensing 73, 12-20 (2012).

49. Zhang, L. et al. Mapping ground surface deformation using temporarily coherent point SAR interferometry: application to Los Angeles Basin. Remote Sensing of Environment 117, 429-439 (2012).

50. Chen, Z., Zhang, L. \& Zhang, G. An improved InSAR image co-registration method for Pairs with relatively big distortions or large incoherent areas. Sensors 16,1519 (2016). 
51. Liang, H., Zhang, L., Ding, X., Lu, Z. \& Li, X. Toward mitigating stratified tropospheric delays in multitemporal InSAR: a quadtree aided joint model. IEEE Transactions on Geoscience and Remote Sensing 57, 291-303 (2019).

52. Zhang, L., Ding, X. \& Lu, Z. Modeling PSInSAR time series without phase unwrapping. IEEE Transactions on Geoscience and Remote Sensing 49, 547-556 (2011).

\section{Acknowledgements}

This study is partially supported by the Vice-Chancellor's One-off Discretionary Fund of The Chinese University of Hong Kong. It is also supported by a GRF 2016/17 (Project No.: 14643816, named "A study of "Local Climate Zone (LCZ)" of Sub-tropical China's Pearl River Delta (PRD) region by using The WUDAPT method for better Comfortable Living and Sustainable Urban Planning) and RGC Germany/Hong Kong Joint Research Scheme 2016/17 (Ref. No.: G-CUHK411/16; Project Title: Towards Urban Planning Strategies to Improve the Thermal Environment in High Density Cities Based on Better Understanding and Extraction of the Urban Morphology in the WUDAPT Framework) of Hong Kong Research Grants Council.

\section{Author contributions}

C.R. and L.Z. designed the study. L.Z., X.L., M.C. and Y.X. performed the research. C.R. and L.Z. wrote the manuscript. M.C., X.L., R.W. and E.N. participated to data analysis and revised this manuscript. All authors discussed results and have given approval to the final version.

\section{Competing interests}

The authors declare no competing interests.

\section{Additional information}

Supplementary information is available for this paper at https://doi.org/10.1038/s41598-019-55444-9.

Correspondence and requests for materials should be addressed to X.L.

Reprints and permissions information is available at www.nature.com/reprints.

Publisher's note Springer Nature remains neutral with regard to jurisdictional claims in published maps and institutional affiliations.

(c) (i) Open Access This article is licensed under a Creative Commons Attribution 4.0 International License, which permits use, sharing, adaptation, distribution and reproduction in any medium or format, as long as you give appropriate credit to the original author(s) and the source, provide a link to the Creative Commons license, and indicate if changes were made. The images or other third party material in this article are included in the article's Creative Commons license, unless indicated otherwise in a credit line to the material. If material is not included in the article's Creative Commons license and your intended use is not permitted by statutory regulation or exceeds the permitted use, you will need to obtain permission directly from the copyright holder. To view a copy of this license, visit http://creativecommons.org/licenses/by/4.0/.

(C) The Author(s) 2019 